\title{
Growth Responses of Hybrid Bluegrass and Tall Fescue as Influenced by Light Intensity and Trinexapac-ethyl
}

\author{
Meghyn Meeks and Ambika Chandra ${ }^{1}$ \\ Texas A\&M AgriLife Research, 17360 Coit Road, Dallas, TX 75252
}

\author{
Ben G. Wherley \\ Department of Soil and Crop Sciences, Texas A\&M University, 370 Olsen \\ Boulevard, 2474 TAMU, College Station, TX 77843
}

Additional index words. shade, low light tolerance, texas $\times$ kentucky bluegrass, leaf elongation, 'DALBG 1201', 'Thermal Blue', 'Kenblue' kentucky bluegrass, 'Rebel Exeda' tall fescue

\begin{abstract}
Interspecific hybrids between texas bluegrass (Poa arachnifera Torr.) and kentucky bluegrass (Poa pratensis L.) are known to exhibit good heat tolerance, which has aided in their adaptation to the warmer climates of the southern United States, but their tolerance to shade has not been investigated. The objectives of this study were to 1$)$ evaluate the growth responses of interspecific bluegrass hybrids $(P$. arachnifera $\times P$. pratensis) in comparison with kentucky bluegrasses and a shade-tolerant cultivar of tall fescue (Festuca arundinacea Schreb.) under full sunlight and shaded environments, 2) identify optimum times to evaluate shade tolerance using the selected growth measurements, 3) calculate the minimum daily light requirements to retain acceptable turfgrass quality, and 4) determine if trinexapac-ethyl (TE) applications enhance hybrid bluegrass quality under shade. Two 10-week greenhouse experiments (late spring and early fall) were conducted in Dallas, TX. Within each of three light environments a randomized complete block design was used to accommodate three replications of eight genotypes treated with and without TE (0 or $0.228 \mathrm{~kg} \cdot \mathrm{ha}^{-1}$ a.i.). Turfgrass quality, leaf elongation rates, clipping dry weights, and percent green cover were measured. Meaningful comparisons were best during the late spring when daily light integrals (DLI) were optimum for healthy plant growth. Shade-tolerant hybrid bluegrasses (DALBG 1201 and TAES 5654) were identified using turfgrass quality and leaf elongation rates. These genotypes exhibited above-acceptable turfgrass quality in all environments, and a reduced leaf elongation rate similar to the tested dwarf-type tall fescue. DLI requirements of DALBG 1201 and TAES 5654 were $\leq 4$ to achieve acceptable quality. TE applications generally did not improve turfgrass quality of genotypes, although leaf elongation rates were significantly reduced in all environments.
\end{abstract}

Shade tolerance is an important factor for selecting turfgrasses for home lawns and recreational facilities. Beard (1973) estimated that $20 \%$ to $25 \%$ of established turfgrass stands are impacted by some type of light restriction. Cool-season $\left(\mathrm{C}_{3}\right)$ turfgrass species generally have lower light compensation points than warm-season $\left(\mathrm{C}_{4}\right)$ turfgrass species. Greater photochemical efficiency at low light intensities contributes to the generally superior shade adaptability of $\mathrm{C}_{3}$ grasses (Kephart and Buxton, 1996). However, only a few of these cool-season species have shown potential for adaptability to the southern or

Received for publication 11 Feb. 2015. Accepted for publication 26 May 2015

This manuscript is in partial fulfillment of a $\mathrm{PhD}$ dissertation for Meghyn Meeks.

We thank Dr. Leah Brilman for providing vegetative plant material of 'Kenblue', and Jim Thomas for his assistance with digital image analysis. We acknowledge Texas A\&M AgriLife Research and NGTurf for partially funding this study.

${ }^{1}$ Corresponding author. E-mail: a-chandra@tamu. edu.
Compact america, compact midnight, and mid-atlantic types display greater tolerances to heat, drought, and shade, but are generally not well adapted to southern environments (Brilman, 2009; Hall, 1996; Morris, 2010). Genes conferring heat and drought tolerances have been introgressed from texas bluegrass (Poa arachnifera Torr.), a native species adapted to the southern plains of the United States (Hitchcock, 1950), into kentucky bluegrass through interspecific hybridization. Some of these hybrid bluegrasses (Poa arachnifera $\times$ Poa pratensis) have demonstrated markedly better performance when compared with kentucky bluegrass and tall fescue (Abraham et al., 2008; Meeks et al., 2015; Su et al., 2007) and have allowed for a greater range of adaptability into more southern environments, but their potential under shaded environments have not been evaluated.

Methods of evaluating the shade tolerance of cool-season grasses include shade structures covered with shadecloth either in the glasshouse or in the field (Burner and West, 2010; Feldhake et al., 1985; Lin et al., 1999; Tan and Qian, 2003; Tegg and Lane, 2004; Watson et al., 1984), under tree canopies (Gardner and Taylor, 2002; Wherley et al., 2005), and in growth chambers (Wood, 1968). In most cases, full sun, moderate $(\approx 50 \%)$, or heavy $(\approx 80 \%)$ shade have been used for comparisons; however, establishment methods and experiment duration vary depending on the objectives and location.

Parameters typically used to characterize turfgrass growth responses under shaded environments include clipping yield, turfgrass quality, and color (Burner and West, 2010; Cockerham et al., 2002; Feldhake et al., 1985; Lin et al., 1999; Watson et al., 1984; Wood, 1968). In cool-season grasses, clipping biomass production has been shown to decrease with decreasing light intensity (Cockerham et al., 2002; Lin et al., 1999; Watson et al., 1984). This may result from increased leaf succulence and narrower, thinner leaves in shade. Leaf elongation on the other hand is one of the most documented shade avoidance mechanisms in genotypes that are intolerant (Beard, 1965; Tan and Qian, 2003; Tegg and Lane, 2004). High leaf elongation rates result in accelerated energy depletion in plant tissue (Qian and Engelke, 1999), increased mowing frequency by the turfgrass manager or homeowner, reduced stand density, and green cover (Gardner and Taylor 2002; Wherley et al., 2013). All of these effects negatively impact turfgrass quality. Plant growth regulators such as flurprimidol or TE have been used to reduce leaf elongation and mowing frequency under full sunlight and shaded conditions (Lickfeldt et al., 2001; Stier et al., 1999; Tan and Qian, 2003), but reports on enhanced turfgrass quality in shade have been inconsistent (Ervin and Koski, 2001; Gardner and Wherley, 2005; Qian et al., 1998; Steinke and Stier, 2003; Stier and Rogers, 2001; Wang et al., 2009).

The objectives of this study were to 1) evaluate the growth responses of interspecific 
bluegrass hybrids in comparison with kentucky bluegrasses and a shade-tolerant cultivar of tall fescue under full sunlight and shaded environments, 2) identify optimum times to evaluate shade tolerance using the selected growth measurements, 3) calculate the minimum daily light requirements to retain acceptable turfgrass quality, and 4) determine if TE applications enhance hybrid bluegrass quality under shade.

\section{Materials and Methods}

Plant materials. Five hybrid bluegrasses (P. arachnifera $\times$ P. pratensis) ['Reveille', 'Thermal Blue', 'DALBG 1201' (PI 671854), TAES 5654, and TAES 5655] were evaluated in comparison with two kentucky bluegrasses (cv. Kenblue and an ecotype, CS\#4, which is a male parent to 'DALBG 1201', TAES 5654 and TAES 5655), and one tall fescue ('Rebel Exeda'). 'Reveille' and 'Thermal Blue' are shade-sensitive and shade-tolerant hybrids, respectively (Morris, 2010, 2013). 'DALBG 1201' (Meeks et al., 2015), TAES 5654, and TAES 5655 are elite hybrids adapted to the southern United States, but have unknown levels of shade tolerance. 'Kenblue' is classified as a common type of kentucky bluegrass sensitive to low-light conditions with increased shoot elongation that reduces stand density and overall turfgrass quality (Morris, 2010; Richardson et al., 2010; Tan and Qian, 2003). 'Rebel Exeda' is an improved turftype tall fescue that has good tolerance to shaded conditions (Wallace et al., 2013).

Plant material was established in $10-\mathrm{cm}$ diameter round pots for 6 months and transplanted into 20 -cm-diameter pots 1 week before initiating each experiment. Soil medium was composed of Sunshine VP mix (Sun Gro Horticulture, Inc., Vancouver, Canada) and 5\% (v:v) sand. Osmocote (14-14 14) (Everris NA, Inc., Dublin, $\mathrm{OH}$ ) fertilizer was incorporated into potting soil at a rate of $7.2 \mathrm{~kg} \cdot \mathrm{m}^{-3}$. All plants were treated uniformly and were trimmed to $5 \mathrm{~cm}$ on the start date of each experiment. Pots were watered 2-3 times weekly for full sunlight treatments, and 1-2 times weekly for the shade treatments. At each irrigation event, irrigation was supplied to fully saturate pots. Drainage ceased and field capacity was achieved usually within 6-10 h following irrigation. Daytime greenhouse temperatures during May-July ranged from 24 to $29{ }^{\circ} \mathrm{C}$, with nighttime temperatures ranging from 18 to $24{ }^{\circ} \mathrm{C}$. From August to October, daytime temperatures ranged from 21 to $33{ }^{\circ} \mathrm{C}$, with nighttime temperatures ranging from 12 to $29^{\circ} \mathrm{C}$.

Experimental design. Two independent experiments were performed for 10 weeks under a glasshouse at the Texas A\&M AgriLife Research Center in Dallas, TX, in 2014. The first experiment occurred from May to July and the second from August to October.

Within each of the three light environments (full sunlight, moderate shade, and heavy shade), treatments were arranged in a randomized complete block design with all possible combinations of genotype and TE (Primo Maxx, Syngenta Co., Ltd., Switzerland). Each genotype $\times$ TE treatment (untreated and $0.228 \mathrm{~kg} \cdot \mathrm{ha}^{-1}$ a.i.) combination was replicated three times within each environment. TE treatments were delivered to plants every 4 weeks using a hand-pumped tank sprayer and fan nozzle. Preventative fungicide applications were tank-mixed with the control and TE treatments using chlorothalonil (DaconilZn, Syngenta Co., Ltd., Switzerland) at $0.79 \mathrm{~kg} \cdot \mathrm{ha}^{-1}$ a.i. and azoxystrobin (Heritage, Syngenta Co., Ltd., Switzerland) at $0.003 \mathrm{~kg} \cdot \mathrm{ha}^{-1}$ a.i. Treatments within each shade environment were re-randomized before each monthly chemical treatment. Shade structures were built to a scale of $274 \mathrm{~cm}$ $\mathrm{L} \times 152 \mathrm{~cm} \mathrm{~W} \times 91 \mathrm{~cm} \mathrm{H}$, and were covered on all sides with black polypropylene shadecloth. Shadecloth density was $40 \%$ (moderate) and $80 \%$ (heavy) to account for the filtered light through the glass roof. A $60-\mathrm{cm}$ opening across the bottom of the north side of the frame allowed for air flow and ease-of-access. Pots were placed $30 \mathrm{~cm}$ inside the north and south edges of the benches to prevent direct transmission of inclined light.

Measurements. Quantum light sensors were used to measure photosynthetic photon flux at the turf canopy level within each light environment. Light data were recorded hourly using a WatchDog 1425 Micro Station

data logger (Spectrum Technologies, Inc., Aurora, IL). Average DLIs were calculated across the duration of each experiment for each light environment using the Specware Software (Spectrum Technologies, Inc).

Leaf tissues were held together in a bunch to determine the three tallest leaves of each pot, from which leaf elongation $\left(\mathrm{cm} \cdot \mathrm{d}^{-1}\right)$ was measured from the height-of-cut to the leaf tip. Average daily leaf elongation rate was obtained by dividing total elongation over the growth period by the number of days since trimming. Pots were only trimmed to the original $5-\mathrm{cm}$ height once every 2 weeks for the purpose of collecting clippings, and leaf elongation measurements were collected before trimming. Collected clippings were dried at $70{ }^{\circ} \mathrm{C}$ for $48 \mathrm{~h}$ (Tan and Qian, 2003). Data for leaf elongation and clipping production were collected every 2 weeks for a total of 5 collection dates in each experiment. An analysis was also performed to determine the minimum number of weeks in shade needed for accurately identifying shade tolerant genotypes from leaf elongation rates. The mean leaf elongation rates for each collection week were regressed against the overall 10-week averages.

Turfgrass quality was assessed during the tenth week of each experiment. Turfgrass quality ratings were assessed on a 1 to 9 scale with a completely brown turf canopy $(=1)$, perfectly dense, uniform, and green canopy

Table 1. Experimental daily light integrals (DLIs) calculated for each light environment.

\begin{tabular}{lccc}
\hline & \multicolumn{3}{c}{ DLI $\left(\mathrm{mol} \cdot \mathrm{m}^{-2} \cdot \mathrm{d}^{-1}\right)^{\mathrm{z}}$} \\
\cline { 2 - 4 } Expt. & Full sunlight & Moderate shade & Heavy shade \\
\hline May-July & $29.9 \pm 10.1$ & $14.7 \pm 5.0$ & $6.3 \pm 2.1$ \\
August-October & $23.5 \pm 6.8$ & $9.9 \pm 3.1$ & $4.1 \pm 1.6$ \\
\hline
\end{tabular}

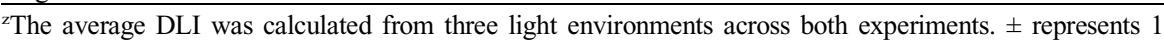
standard deviation. Averages can be used to determine the percentage of shade density in comparison with the full sunlight. For example, for May-July, $1-(14.7 / 29.9) \approx 50 \%$ shade and $1-(6.3 / 29.9) \approx 80 \%$ shade.

Table 2. Final turfgrass quality of individual genotypes under full sunlight, moderate shade, and heavy shade environments.

\begin{tabular}{|c|c|c|c|c|c|c|}
\hline \multirow[b]{3}{*}{ Genotype } & \multicolumn{6}{|c|}{ Final turfgrass quality $(1-9 \text { scale })^{\mathrm{z}}$} \\
\hline & \multicolumn{2}{|c|}{ Full sunlight } & \multicolumn{2}{|c|}{ Moderate shade } & \multicolumn{2}{|c|}{ Heavy shade } \\
\hline & Expt. 1 & Expt. 2 & Expt. 1 & Expt. 2 & Expt. 1 & Expt. 2 \\
\hline Rebel Exeda & $7.7 \mathrm{ab}$ & $7.0 \mathrm{ab}$ & $6.7 \mathrm{a}$ & 5.0 abc & $5.3 \mathrm{ab}$ & $2.7 \mathrm{~b}^{\dagger}$ \\
\hline Kenblue & $5.7 \mathrm{c}$ & $4.7 \mathrm{c}$ & $4.0 \mathrm{~d}$ & $3.3 \mathrm{c}$ & $2.7 \mathrm{~b}$ & $2.3 \mathrm{~b}$ \\
\hline $\mathrm{CS} \# 4$ & $7.7 \mathrm{ab}$ & $7.0 \mathrm{ab}$ & $5.7 \mathrm{abc}$ & $4.3 \mathrm{abc}^{\dagger}$ & $4.7 \mathrm{ab}$ & $2.0 \mathrm{~b}^{\dagger}$ \\
\hline Thermal Blue & $6.7 \mathrm{bc}$ & $6.0 \mathrm{bc}$ & $4.3 \mathrm{~cd}$ & $3.7 \mathrm{bc}$ & $3.7 \mathrm{ab}$ & $2.7 \mathrm{~b}$ \\
\hline Reveille & $5.7 \mathrm{c}^{\dagger}$ & $8.0 \mathrm{ab}$ & $5.0 \mathrm{bcd}$ & $4.0 \mathrm{abc}$ & $3.7 \mathrm{ab}$ & $4.3 \mathrm{ab}$ \\
\hline DALBG 1201 & $8.7 \mathrm{a}$ & $9.0 \mathrm{a}$ & $7.0 \mathrm{a}$ & $7.3 \mathrm{a}$ & $5.7 \mathrm{a}$ & $6.0 \mathrm{a}$ \\
\hline TAES 5654 & $9.0 \mathrm{a}$ & $9.0 \mathrm{a}$ & $6.3 \mathrm{ab}$ & $7.0 \mathrm{ab}$ & $5.3 \mathrm{ab}$ & $5.7 \mathrm{a}$ \\
\hline TAES 5655 & $6.3 \mathrm{bc}$ & $7.0 \mathrm{ab}$ & $5.0 \mathrm{bcd}$ & $6.0 \mathrm{abc}$ & $2.7 \mathrm{~b}$ & $4.3 \mathrm{ab}$ \\
\hline
\end{tabular}

\section{ANOVA}

Experiment ${ }^{y}$

Genotype $^{\mathrm{x}}$

Experiment $\times$ genotype $^{\mathrm{w}}$

\begin{tabular}{|c|c|c|c|c|c|}
\hline \multicolumn{2}{|c|}{ NS } & \multicolumn{2}{|c|}{ NS } & \multicolumn{2}{|c|}{ NS } \\
\hline$* * * *$ & $* * * *$ & $* * * *$ & $* *$ & $* *$ & $* * *$ \\
\hline & & & & & \\
\hline
\end{tabular}

zTurfgrass quality ratings were assessed on a 1-9 scale $(1=$ brown/tan or dead; $5=$ minimum acceptable quality).

'Differences between experiments were determined using Student's $t$ test at a significance level of $P \leq$ 0.05 .

${ }^{x}$ Genotype means within columns were separated using Tukey's honestly significant difference test at a significance level of $P \leq 0.05$. Means followed by the same letter are not significantly different.

${ }^{w}$ For each genotype, significant mean differences between experiments are indicated by a dagger $(\dagger)$. $\mathrm{NS}, *, * *, * * *, * * *$ Nonsignificant or significant at $P \leq 0.05,0.01,0.001,0.0001$, respectively. ANOVA $=$ analysis of variance. 
Table 3. The effect of trinexapac-ethyl (TE) on turfgrass quality of individual genotypes under full sunlight, moderate shade, and heavy shade.

\begin{tabular}{|c|c|c|c|c|c|c|c|c|c|c|c|c|}
\hline \multirow{2}{*}{ Genotype } & \multicolumn{12}{|c|}{ Final turfgrass quality $(1-9 \text { scale })^{z}$} \\
\hline & \multicolumn{4}{|c|}{ Full sunlight } & \multicolumn{4}{|c|}{ Moderate shade } & \multicolumn{4}{|c|}{ Heavy shade } \\
\hline Rebel Exeda & 7.3 & 7.5 & $0.2^{\mathrm{NS}}$ & 3 & 5.8 & 6.5 & $0.7^{\mathrm{NS}}$ & 12 & 4.0 & 3.8 & $-0.2^{\mathrm{NS}}$ & -5 \\
\hline CS\#4 & 7.3 & 7.2 & $-0.1^{\mathrm{NS}}$ & -1 & 5.0 & 5.7 & $0.7^{\mathrm{NS}}$ & 14 & 3.3 & 4.0 & $0.7^{\mathrm{NS}}$ & 21 \\
\hline Thermal Blue & 6.3 & 6.5 & $0.2^{\mathrm{NS}}$ & 3 & 4.0 & 5.2 & $1.2^{*}$ & 30 & 3.2 & 2.7 & $-0.5^{\mathrm{NS}}$ & -16 \\
\hline Reveille & 6.8 & 6.5 & $-0.3^{\mathrm{NS}}$ & -4 & 4.5 & 4.7 & $0.2^{\mathrm{NS}}$ & 4 & 4.0 & 1.8 & $-2.2^{*}$ & -55 \\
\hline DALBG 1201 & 8.8 & 8.2 & $-0.6^{\mathrm{NS}}$ & -7 & 7.2 & 6.7 & $-0.5^{*}$ & -7 & 5.8 & 5.5 & $-0.3^{\mathrm{NS}}$ & -5 \\
\hline \multicolumn{13}{|l|}{ ANOVA } \\
\hline Experiment $^{\mathrm{w}}$ & \multicolumn{4}{|c|}{ NS } & \multicolumn{4}{|c|}{ NS } & \multicolumn{4}{|c|}{$* *$} \\
\hline Treatment $^{v}$ & \multicolumn{4}{|c|}{ NS } & \multirow{2}{*}{\multicolumn{4}{|c|}{ NS }} & \multicolumn{4}{|c|}{ NS } \\
\hline Experiment $\times$ treatment & \multicolumn{4}{|c|}{ NS } & & & & NS & \multicolumn{4}{|c|}{ NS } \\
\hline
\end{tabular}

${ }^{2}$ Turfgrass quality ratings were assessed on a $1-9$ scale $(1=$ brown/tan or dead; $5=$ minimum acceptable quality).

${ }^{y}$ Mean differences for turfgrass quality were calculated by subtracting mean control ratings from mean TE ratings.

${ }^{\mathrm{x}}$ The percentage in change was calculated by dividing the mean difference over the control mean and multiplying by 100 .

wTurfgrass quality between experiments were determined using Student's $t$ test at a significance level of $P \leq 0.05$. Data were pooled for all environments.

'Differences in mean turfgrass quality between the control and TE treatment for each individual genotype were determined using Student's $t$ test at a significance level of $P \leq 0.05$.

Ns, *Nonsignificant or significant at $P \leq 0.05$.

$(=9)$, and minimally acceptable quality $(=5)$. The overall mean turfgrass quality for each genotype in the three light environments was regressed against the calculated DLI averages for each light environment to determine minimum DLI for acceptable turfgrass quality (Bunnell et al., 2005).

Final green cover $(\%)$ was assessed at week 10 using digital images of each pot and SigmaScan Pro Version 5.0 (Systat, Inc., Richmond, CA) with the Turf Analysis 1.2 macro (Karcher and Richardson, 2005). Threshold hue (16-47) and saturation (3856) settings were adjusted to select for yellow or dead tissue. Values were subtracted from 100 to obtain percent green cover. Final percent green cover values were plotted against average leaf elongation rates for full sunlight, moderate shade, and heavy shade to better understand the relationship between the two parameters for shade screening evaluation.

Analysis of variance (ANOVA) and Pearson's correlation analyses were performed using JMP 10 (SAS Institute Inc., 2012), with shade environments analyzed independently. Fixed factors included experiment, TE treatment, and genotype. Replications were treated as random variables. Collection week was only considered a fixed effect for leaf elongation. Experimental differences were determined using Student's $t$ test at a significance level of $P \leq 0.05$. Mean separation was performed using Tukey's honestly significant difference test at a significance level of $P \leq 0.05$.

\section{Results and Discussion}

The average DLIs for the two experiments are shown in Table 1. DLIs for the full sunlight environment were $29.9 \pm 10.1$ and $23.5 \pm 6.8 \mathrm{~mol} \cdot \mathrm{m}^{-2} \cdot \mathrm{d}^{-1}$ which were $\approx 89.3 \%$ and $76.5 \%$ of ambient DLI for experiments 1 and 2 , respectively. The shadecloth used for moderate shade resulted in DLIs of $14.7 \pm 5.0$

Table 4. Final percent green cover of individual genotypes under full sunlight, moderate shade, and heavy shade environments.

\begin{tabular}{|c|c|c|c|c|c|c|}
\hline \multirow[b]{3}{*}{ Genotype } & \multicolumn{6}{|c|}{ Final green cover $(\%)^{\mathrm{z}}$} \\
\hline & \multicolumn{2}{|c|}{ Full sunlight } & \multicolumn{2}{|c|}{ Moderate shade } & \multicolumn{2}{|c|}{ Heavy shade } \\
\hline & Expt. 1 & $\overline{\text { Expt. } 2}$ & Expt. 1 & $\overline{\text { Expt. } 2}$ & Expt. 1 & Expt. 2 \\
\hline Rebel Exeda & $99.8 \mathrm{a}$ & $95.0 \mathrm{ab}$ & $99.0 \mathrm{a}$ & $84.6 \mathrm{ab}^{\dagger}$ & $96.2 \mathrm{a}$ & $67.2 \mathrm{c}^{\dagger}$ \\
\hline Kenblue & $96.3 \mathrm{~b}$ & $85.6 \mathrm{bc}^{\dagger}$ & $87.3 \mathrm{~b}$ & $76.5 \mathrm{ab}^{\dagger}$ & $87.4 \mathrm{~b}$ & $82.5 \mathrm{ab}$ \\
\hline CS\#4 & $99.2 \mathrm{a}$ & $96.6 \mathrm{a}$ & $88.3 \mathrm{~b}$ & $68.9 \mathrm{~b}^{\dagger}$ & $77.5 \mathrm{~b}$ & $69.8 \mathrm{c}$ \\
\hline Thermal Blue & $96.3 \mathrm{~b}$ & $85.4 \mathrm{c}$ & $88.3 \mathrm{~b}$ & $79.5 \mathrm{ab}^{\dagger}$ & $88.2 \mathrm{~b}$ & $75.8 \mathrm{bc}^{\dagger}$ \\
\hline Reveille & $99.5 \mathrm{a}$ & $94.9 \mathrm{abc}$ & $94.6 \mathrm{a}$ & $74.6 \mathrm{ab}$ & $91.0 \mathrm{ab}$ & $82.9 \mathrm{ab}$ \\
\hline DALBG 1201 & $99.6 \mathrm{a}$ & $99.5 \mathrm{a}$ & $98.6 \mathrm{a}$ & $93.1 \mathrm{a}$ & $93.8 \mathrm{ab}$ & $90.5 \mathrm{a}$ \\
\hline TAES 5654 & $99.7 \mathrm{a}$ & $99.5 \mathrm{a}$ & $96.0 \mathrm{a}$ & $92.3 \mathrm{a}$ & $92.1 \mathrm{ab}$ & $89.2 \mathrm{a}$ \\
\hline TAES 5655 & $99.1 \mathrm{a}$ & $95.7 \mathrm{a}$ & $94.6 \mathrm{a}$ & $86.1 \mathrm{ab}$ & $89.2 \mathrm{~b}$ & $85.3 \mathrm{ab}$ \\
\hline \multicolumn{7}{|l|}{ ANOVA } \\
\hline Experiment $\mathrm{y}^{\mathrm{y}}$ & \multicolumn{2}{|c|}{$* * * *$} & \multicolumn{2}{|c|}{$* * * *$} & \multicolumn{2}{|c|}{$* * * *$} \\
\hline Genotype $^{\mathrm{x}}$ & $* * * *$ & $* * *$ & $* * * *$ & ** & $* * * *$ & $* * * *$ \\
\hline Experiment $\times$ genotype $^{\mathrm{w}}$ & \multicolumn{2}{|c|}{$* * *$} & \multicolumn{2}{|c|}{ NS } & \multicolumn{2}{|c|}{$* * * *$} \\
\hline
\end{tabular}

${ }^{2}$ Final green cover was assessed as a percentage using digital image analysis during the final week of each experiment.

y Differences between experiments were determined using Student's $t$ test at a significance level of $P \leq$ 0.05 .

${ }^{\mathrm{x}}$ Genotype means within columns were separated using Tukey's honestly significant difference test at a significance level of $P \leq 0.05$. Means followed by the same letter are not significantly different.

${ }^{\text {w}}$ For each genotype, significant mean differences between experiments are indicated by a dagger $(\dagger)$. Ns, $* * *, * * *, * * *$ Nonsignificant or significant at $P \leq 0.05,0.01,0.001,0.0001$, respectively. ANOVA $=$ analysis of variance.

and $9.9 \pm 3.0 \mathrm{~mol} \cdot \mathrm{m}^{-2} \cdot \mathrm{d}^{-1}$, or $\approx 50 \%$ and $60 \%$ of ambient DLI for experiments 1 and 2, respectively. The shadecloth used for heavy shade produced DLIs of $6.3 \pm 2.1$ and $4.1 \pm$ $1.6 \mathrm{~mol} \cdot \mathrm{m}^{-2} \cdot \mathrm{d}^{-1}$, or $\approx 80 \%$ of ambient DLI in both experiments. Despite the similarity in calculated DLIs, the average photoperiod during experiment 1 was nearly $2 \mathrm{~h}$ longer and more variable than experiment 2. Environmental factors such as presence and duration of cloud cover in experiment 1 may have contributed to the lower-than-expected DLI and greater variability. Additionally, this 2 hour difference may have had an impact on plant growth characteristics.

Differences in DLI between seasons have been reported to influence growth and performance of genotypes under shade environments
(Lin et al., 1999). Based on the calculated mean DLIs for each light environment $x$ experiment, mean DLIs fell below critical levels for kentucky bluegrass (Cockerham et al., 2002) and other cool-season grasses (Beard 1973) under both moderate and heavy shade for experiment 2, indicating that early fall conditions were not ideal for evaluating bluegrasses for shade tolerance. Additionally, greenhouse temperatures were slightly higher during the period of August to October, which could have contributed to higher rates of photorespiration in the latter experiment (Su et al., 2007).

Turfgrass quality. Within each environment, no significant differences were calculated between experiments, but significant experiment $\times$ genotype interactions indicated 
that some genotypes performed differently between the two experiments (Table 2). In particular, 'Reveille' rated significantly higher in full sunlight during experiment 2, whereas significantly lower ratings were observed for the shade-tolerant CS\#4 and 'Rebel Exeda' maintained in shade during experiment 2 . The commercial hybrid, 'Thermal Blue', did not display acceptable turfgrass quality in any shade environment for either experiment, suggesting that it lacks good shade tolerance. In both experiments, the shade-sensitive kentucky bluegrass control, 'Kenblue', failed to attain acceptable quality ratings within either shade treatment. 'Reveille' also exhibited poor shade tolerance, with acceptable shade quality (5.0) noted only under moderate shade during experiment 1 . Lower quality ratings were observed for the majority of genotypes during experiment 2 , likely due to insufficient light levels for these genotypes. Hybrid bluegrasses 'DALBG 1201' and TAES 5654, however, exhibited superior turfgrass quality relative to the commercial varieties within both shade environments.

In evaluating the effects of TE in the study, the overall effects of experiment, treatment, and experiment $\times$ treatment were not significant (Table 3). However, a significant effect of experiment for heavy shade likely attributed to the different total DLIs between the two studies. Additionally, TE failed to impact quality of the majority of genotypes, regardless of the light environment. Our results for effects of TE on turf quality in heavy shade are consistent with Gardner and Wherley (2005), who reported turfgrass quality of cool-season grasses including tall fescue was not enhanced with TE treatment under $\approx 90 \%$ shade levels. Turfgrass quality is a factor of color, texture, density, and uniformity; hence the combined effect of reduced growth and stand density from $\mathrm{TE}$ application under shade may have contributed to the reduction in overall turfgrass quality ratings (data not shown). Based on our results, TE does not appear to be a useful tool for mitigating hybrid bluegrass turf quality loss in shade.

Final percent green cover. It was observed that green cover was statistically highest for experiment 1 across all environments; however, there was a genotype $\times$ experiment interaction noted within moderate shade (Table 4). Although hybrid bluegrasses showed superior cover relative to kentucky bluegrass in moderate shade, the two species did not differ under heavy shade. Final percent green cover of four out the five hybrid bluegrass genotypes did not differ from one another or 'Rebel Exeda' in either full sunlight or shade environments.

The ability of a grass to retain green color and density while laterally growing under shade contributes to greater levels of final percent green cover. This has been used both in cool- and warm-season grasses for longterm studies (Gardner and Taylor, 2002; Wherley et al., 2013). This study was relatively short-term, designed to evaluate the growth responses of breeding lines for advancement. Considering the lack of significant differences detected and short duration of the study, we did not find final percent green cover to be useful for identifying shade-tolerant hybrid bluegrasses.

Cumulative clipping production. In this study, experiment, genotype, and experiment $\times$ genotype effects were all significant factors for cumulative clipping production (Table 5). In general, cumulative clipping production was highest during experiment 1 for all

Table 5. Cumulative clipping production of individual genotypes under full sunlight, moderate shade, and heavy shade environments.

\begin{tabular}{|c|c|c|c|c|c|c|}
\hline \multirow[b]{3}{*}{ Genotype } & \multicolumn{6}{|c|}{ Cumulative clipping production $(\mathrm{g})^{\mathrm{z}}$} \\
\hline & \multicolumn{2}{|c|}{ Full sunlight } & \multicolumn{2}{|c|}{ Moderate shade } & \multicolumn{2}{|c|}{ Heavy shade } \\
\hline & Expt. 1 & $\overline{\text { Expt. } 2}$ & Expt. 1 & Expt. 2 & Expt. 1 & $\overline{\text { Expt. } 2}$ \\
\hline Rebel Exeda & $11.6 \mathrm{ab}$ & $4.3 \mathrm{~b}$ & $3.8 \mathrm{~cd}$ & $2.3 \mathrm{a}^{\dagger}$ & $2.0 \mathrm{bc}$ & $1.4 \mathrm{ab}$ \\
\hline Kenblue & $16.7 \mathrm{a}$ & $9.5 \mathrm{ab}^{\dagger}$ & $5.6 \mathrm{bc}$ & $2.4 \mathrm{a}^{\dagger}$ & $1.7 \mathrm{bc}$ & $0.6 \mathrm{~b}$ \\
\hline CS\#4 & $14.2 \mathrm{a}$ & $9.2 \mathrm{ab}$ & $10.0 \mathrm{a}$ & $2.8 \mathrm{a}^{\dagger}$ & $4.6 \mathrm{a}$ & $1.4 \mathrm{ab}^{\dagger}$ \\
\hline Thermal Blue & $16.0 \mathrm{a}$ & $10.7 \mathrm{a}^{\dagger}$ & $7.9 \mathrm{ab}$ & $2.5 \mathrm{a}^{\dagger}$ & $2.4 \mathrm{~b}$ & $1.6 \mathrm{ab}^{\dagger}$ \\
\hline Reveille & $5.9 \mathrm{~b}$ & $5.4 \mathrm{~b}$ & $3.6 \mathrm{~cd}$ & $2.7 \mathrm{a}$ & $1.2 \mathrm{bc}$ & $1.0 \mathrm{ab}$ \\
\hline DALBG 1201 & $6.1 \mathrm{~b}$ & $6.2 \mathrm{ab}$ & $4.6 \mathrm{~cd}$ & $2.8 \mathrm{a}^{\dagger}$ & $2.4 \mathrm{~b}$ & $1.7 \mathrm{ab}^{\dagger}$ \\
\hline TAES 5654 & $6.1 \mathrm{~b}$ & $4.6 \mathrm{~b}^{\dagger}$ & $3.4 \mathrm{~cd}$ & $3.1 \mathrm{a}$ & $1.7 \mathrm{bc}$ & $2.0 \mathrm{a}$ \\
\hline TAES 5655 & $7.1 \mathrm{~b}$ & $5.6 \mathrm{ab}$ & $2.9 \mathrm{~d}$ & $3.7 \mathrm{a}$ & $1.0 \mathrm{c}$ & $1.0 \mathrm{ab}$ \\
\hline
\end{tabular}

ANOVA

Experiment ${ }^{\mathrm{y}}$

Genotype $^{\mathrm{x}}$

Experiment $\times$ genotype $^{\mathrm{w}}$
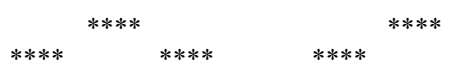

NS

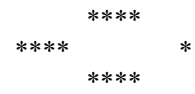

${ }^{2}$ Clippings were collected every 2 weeks during each experiment. Genotypes were compared by their cumulative clipping production in each experiment.

${ }^{\mathrm{y}}$ Differences between experiments were determined using Student's $t$ test at a significance level of $P \leq$ 0.05 .

${ }^{\mathrm{x}}$ Genotype means within columns were separated using Tukey's honestly significant difference test at a significance level of $P \leq 0.05$. Means followed by the same letter are not significantly different.

${ }^{w}$ For each genotype, significant mean differences between experiments are indicated by a dagger $(\dagger)$. Ns, $* * *, * * * * * *$ Nonsignificant or significant at $P \leq 0.05,0.01,0.001,0.0001$, respectively.

ANOVA $=$ analysis of variance.

Table 6. Daily leaf elongation rate of individual genotypes under full sunlight, moderate shade, and heavy shade environments.

\begin{tabular}{|c|c|c|c|c|c|c|}
\hline \multirow[b]{3}{*}{ Genotype } & \multicolumn{6}{|c|}{ Daily leaf elongation rate $\left(\mathrm{cm} \cdot \mathrm{d}^{-1}\right)^{\mathrm{z}}$} \\
\hline & \multicolumn{2}{|c|}{ Full sunlight } & \multicolumn{2}{|c|}{ Moderate shade } & \multicolumn{2}{|c|}{ Heavy shade } \\
\hline & Expt. 1 & Expt. 2 & Expt. 1 & Expt. 2 & Expt. 1 & Expt. 2 \\
\hline Rebel Exeda & $0.7 \mathrm{~d}$ & $0.7 \mathrm{~d}$ & $0.8 \mathrm{de}$ & $0.8 \mathrm{~d}$ & $0.8 \mathrm{c}$ & $0.5 \mathrm{~d}^{\dagger}$ \\
\hline Kenblue & $1.8 \mathrm{a}$ & $1.6 \mathrm{a}$ & $1.8 \mathrm{a}$ & $1.6 \mathrm{a}^{\dagger}$ & $1.5 \mathrm{a}$ & $1.0 \mathrm{~b}^{\dagger}$ \\
\hline CS\#4 & $1.0 \mathrm{c}$ & $1.1 \mathrm{c}$ & $1.1 \mathrm{c}$ & $1.0 \mathrm{c}$ & $1.1 \mathrm{~b}$ & $0.8 \mathrm{bc}^{\dagger}$ \\
\hline Thermal Blue & $1.3 \mathrm{~b}$ & $1.4 \mathrm{~b}$ & $1.5 \mathrm{~b}$ & $1.3 \mathrm{~b}^{\dagger}$ & $1.3 \mathrm{ab}$ & $1.3 \mathrm{a}$ \\
\hline Reveille & $1.0 \mathrm{c}$ & $0.7 \mathrm{~d}^{\dagger}$ & $1.0 \mathrm{~cd}$ & $0.8 \mathrm{~d}^{\dagger}$ & $0.7 \mathrm{c}$ & $0.6 \mathrm{~cd}$ \\
\hline DALBG 1201 & $0.7 \mathrm{~d}$ & $0.7 \mathrm{~d}$ & $0.8 \mathrm{e}$ & $0.7 \mathrm{~d}^{\dagger}$ & $0.7 \mathrm{c}$ & $0.6 \mathrm{~cd}$ \\
\hline TAES 5654 & $0.6 \mathrm{~d}$ & $0.6 \mathrm{~d}$ & $0.7 \mathrm{e}$ & $0.7 \mathrm{~d}$ & $0.6 \mathrm{c}$ & $0.7 \mathrm{~cd}$ \\
\hline TAES 5655 & $0.9 \mathrm{c}$ & $1.0 \mathrm{c}$ & 0.9 cde & $1.0 \mathrm{c}^{\dagger}$ & $0.7 \mathrm{c}$ & $0.8 \mathrm{bcd}$ \\
\hline
\end{tabular}

ANOVA

Experiment ${ }^{\mathrm{y}}$

Genotype $^{\mathrm{x}}$

Experiment $\times$ genotype $^{\mathrm{w}}$

Week

Experiment $\times$ week

Week $\times$ genotype

Experiment $\times$ genotype $\times$ week
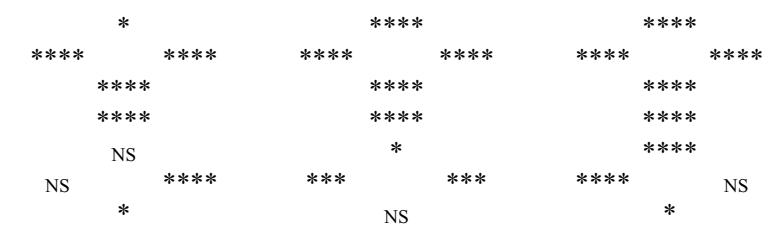

${ }^{2}$ Leaf elongation was measured from the three tallest leaves every 2 weeks in each experiment and divided by the number of days since trimming to determine the average daily leaf elongation rate. The experiment averages across all collection days are presented.

'Differences between experiments were determined using Student's $t$ test at a significance level of $P \leq$ 0.05 .

${ }^{x}$ Genotype means within columns were separated using Tukey's honestly significant difference test at a significance level of $P \leq 0.05$. Means followed by the same letter are not significantly different.

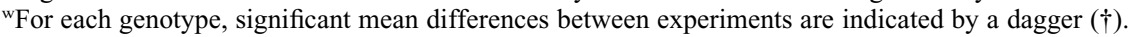
NS, *,**,*******Nonsignificant or significant at $P \leq 0.05,0.01,0.001,0.0001$, respectively.

ANOVA $=$ analysis of variance. 
Table 7. The effect of trinexapac-ethyl (TE) on leaf elongation rate of individual genotypes under full sunlight, moderate shade, and heavy shade.

\begin{tabular}{|c|c|c|c|c|c|c|c|c|c|c|c|c|c|}
\hline & \multirow{2}{*}{ Genotype } & \multicolumn{12}{|c|}{ Daily leaf elongation rate $\left(\mathrm{cm} \cdot \mathrm{d}^{-1}\right)$} \\
\hline & & \multicolumn{4}{|c|}{ Full Sunlight } & \multicolumn{4}{|c|}{ Moderate Shade } & \multicolumn{4}{|c|}{ Heavy Shade } \\
\hline \multirow[t]{5}{*}{ Experiment 1} & Rebel Exeda & 0.7 & 0.5 & $-0.2^{*}$ & -29 & 0.8 & 0.4 & $-0.4^{*}$ & -50 & 0.8 & 0.3 & $-0.5^{*}$ & -63 \\
\hline & CS\#4 & 1.0 & 0.6 & $-0.4 *$ & -40 & 1.1 & 0.4 & $-0.7 *$ & -64 & 1.1 & 0.3 & $-0.8^{*}$ & -73 \\
\hline & Thermal Blue & 1.3 & 0.7 & $-0.6^{*}$ & -46 & 1.5 & 0.6 & $-0.9 *$ & -60 & 1.3 & 0.4 & $-0.9^{*}$ & -69 \\
\hline & Reveille & 1.0 & 0.6 & $-0.4^{*}$ & -40 & 1.0 & 0.4 & $-0.6^{*}$ & -60 & 0.7 & 0.2 & $-0.5^{*}$ & -71 \\
\hline & DALBG 1201 & 0.7 & 0.4 & $-0.3^{*}$ & -43 & 0.8 & 0.3 & $-0.5^{*}$ & -63 & 0.7 & 0.2 & $-0.5^{*}$ & -71 \\
\hline \multirow[t]{7}{*}{ Experiment 2} & Rebel Exeda & 0.7 & 0.5 & $-0.2^{*}$ & -29 & 0.8 & 0.4 & $-0.4^{*}$ & -50 & 0.5 & 0.1 & $-0.4^{*}$ & -80 \\
\hline & Kenblue & 1.6 & 0.9 & $-0.7^{*}$ & -44 & 1.6 & 0.6 & $-1.0^{*}$ & -63 & 1.0 & 0.1 & $-0.9^{*}$ & -90 \\
\hline & CS\#4 & 1.1 & 0.6 & $-0.5^{*}$ & -45 & 1.0 & 0.3 & $-0.7 *$ & -70 & 0.8 & 0.2 & $-0.6^{*}$ & -75 \\
\hline & Thermal Blue & 1.4 & 0.7 & $-0.7^{*}$ & -50 & 1.3 & 0.4 & $-0.9^{*}$ & -69 & 1.3 & 0.3 & $-1.0^{*}$ & -77 \\
\hline & Reveille & 0.7 & 0.5 & $-0.2^{*}$ & -29 & 0.8 & 0.2 & $-0.6^{*}$ & -75 & 0.6 & 0.2 & $-0.4^{*}$ & -67 \\
\hline & DALBG 1201 & 0.7 & 0.3 & $-0.4 *$ & -57 & 0.7 & 0.2 & $-0.5^{*}$ & -71 & 0.6 & 0.2 & $-0.4^{*}$ & -67 \\
\hline & TAES 5654 & 0.6 & 0.3 & $-0.3^{*}$ & -50 & 0.7 & 0.2 & $-0.5^{*}$ & -71 & 0.7 & 0.1 & $-0.6^{*}$ & -86 \\
\hline
\end{tabular}

\section{ANOVA}

Experiment $^{\mathrm{x}}$

Treatment ${ }^{\mathrm{w}}$

Experiment $\times$ treatment

**
$* * * *$
NS

$\begin{array}{ll}\mathrm{NS} \\ * * * * & \mathrm{NS} \\ * * * * & \mathrm{NS}\end{array}$

${ }^{2}$ Mean differences for average daily leaf elongation rates were calculated by subtracting mean control ratings from mean TE ratings.

${ }^{\mathrm{y}}$ The percentage in change was calculated by dividing the mean difference over the control mean and multiplying by 100 .

${ }^{x}$ Leaf elongation rate differences between experiments were determined using Student's $t$ test at a significance level of $P \leq 0.05$. Experiments were significantly different in all environments, so data were not pooled.

"Differences in mean leaf elongation rate between the control and TE treatment for each individual genotype were determined using Student's $t$ test at a significance level of $P \leq 0.05$.

NS, ***,***,***Nonsignificant or significant at $P \leq 0.05,0.01,0.001,0.0001$, respectively.

production. Additionally, the cumulative clipping production data under heavy shade showed that similar amounts of clippings were obtained between genotypes that were found to be tolerant ('Rebel Exeda') and intolerant ('Kenblue') of shade.

As was expected, the effect of TE was significant in reducing clipping production in all environments (data not shown). However, under full sunlight, the effect on 'Rebel Exeda' was not significant as it was for all other genotypes. These results agree with those presented by Stier and Rogers (2001) and Tan and Qian (2003) for kentucky bluegrasses.

The observation that overall clipping production decreased with increasing shade is consistent with previous research (Cockerham et al., 2002; Lin et al., 1999; Watson et al., 1984), and is likely due to altered leaf morphology and general decline in turf density. However, meaningful relationships between clipping production and shade tolerance were difficult to infer in this study due to similarities between shade sensitive and tolerant checks in all environments. This result was congruent to Busey and Davis (1991) on warm-season grasses.

Daily leaf elongation rate. In this study, significant differences in leaf elongation rate due to experiment, genotype, experiment $\times$ genotype, collection week, and collection week $\times$ genotype were detected (Table 6 ). For all environments, growth rates were significantly greater during experiment 1 , although experimental differences only ranged from 0.02 to $0.135 \mathrm{~cm} \cdot \mathrm{d}^{-1}$ in each environment. Individually, hybrid TAES 5655 had a minimal increase in leaf elongation rate during

Table 8. Daily leaf elongation rates calculated every two weeks during experiment 1 under moderate shade.

\begin{tabular}{|c|c|c|c|c|c|c|}
\hline \multirow[b]{3}{*}{ Genotype $^{z}$} & \multicolumn{6}{|c|}{ Daily leaf elongation rate $\left(\mathrm{cm} \cdot \mathrm{d}^{-1}\right)$} \\
\hline & \multicolumn{5}{|c|}{ Collection week } & \multirow[b]{2}{*}{ Avg } \\
\hline & 2 & 4 & 6 & 8 & 10 & \\
\hline Rebel Exeda & $0.6 \mathrm{~d}$ & $0.8 \mathrm{e}$ & $1.0 \mathrm{c}$ & $1.0 \mathrm{bc}$ & $0.8 \mathrm{c}$ & $0.8 \mathrm{~d}$ \\
\hline Kenblue & $1.8 \mathrm{a}$ & $1.8 \mathrm{a}$ & $2.0 \mathrm{a}$ & $2.0 \mathrm{a}$ & $1.5 \mathrm{a}$ & $1.8 \mathrm{a}$ \\
\hline CS\#4 & $0.7 \mathrm{~d}$ & $1.2 \mathrm{c}$ & $1.4 \mathrm{~b}$ & $1.2 \mathrm{~b}$ & $0.9 \mathrm{bc}$ & $1.1 \mathrm{c}$ \\
\hline Thermal Blue & $1.3 \mathrm{~b}$ & $1.5 \mathrm{~b}$ & $1.8 \mathrm{a}$ & $1.6 \mathrm{a}$ & $1.5 \mathrm{ab}$ & $1.5 \mathrm{~b}$ \\
\hline Reveille & $1.1 \mathrm{bc}$ & $1.1 \mathrm{~cd}$ & $1.1 \mathrm{c}$ & $1.0 \mathrm{bc}$ & $0.7 \mathrm{c}$ & $1.0 \mathrm{c}$ \\
\hline DALBG 1201 & $0.7 \mathrm{~d}$ & $0.9 \mathrm{de}$ & $0.9 \mathrm{c}$ & $0.8 \mathrm{bc}$ & $0.7 \mathrm{c}$ & $0.8 \mathrm{~d}$ \\
\hline TAES 5654 & $0.6 \mathrm{~d}$ & $0.7 \mathrm{e}$ & $0.9 \mathrm{c}$ & $0.7 \mathrm{c}$ & $0.5 \mathrm{c}$ & $0.7 \mathrm{~d}$ \\
\hline TAES 5655 & $0.9 \mathrm{~cd}$ & $1.1 \mathrm{~cd}$ & $1.1 \mathrm{c}$ & $0.7 \mathrm{c}$ & $0.7 \mathrm{c}$ & $0.9 \mathrm{c}$ \\
\hline Model $R^{2}$ & 0.86 & 0.96 & 0.97 & 0.94 & 0.91 & - \\
\hline$r^{\mathrm{x}}$ & 0.93 & 0.98 & 0.98 & 0.97 & 0.95 & - \\
\hline$P$ value & 0.0008 & $<0.0001$ & $<0.0001$ & $<0.0001$ & 0.0003 & \\
\hline
\end{tabular}

${ }^{\mathrm{z}}$ Genotype means were separated using Tukey's honestly significant difference test at a significance level of $P \leq 0.05$.

${ }^{y}$ Same as Table 6 for moderate shade experiment 1.

${ }^{\mathrm{x}}$ Genotype weekly averages were regressed against the overall experiment genotype averages.

$R^{2}=$ Coefficient of determination; $r=$ Pearson's correlation coefficient.

experiment 2. The leaf elongation rate of the dwarf-type tall fescue, 'Rebel Exeda', was statistically similar to 'DALBG 1201' and TAES 5654 under full sunlight. With the exception of 'Thermal Blue', under moderate and heavy shade, four out of five hybrid bluegrasses exhibited statistically similar leaf elongation rates to 'Rebel Exeda'. Under moderate shade, 'DALBG 1201' and TAES 5654 were significantly lower than 'Reveille' and TAES 5655.

The application of TE resulted in significant reductions in leaf elongation rate for all genotypes in all environments and experiments (Table 7). The observed reductions were greatest under heavy shade $(60 \%-73 \%)$, followed by moderate shade $(50 \%-64 \%)$, and full sunlight $(29 \%-46 \%)$ in experiment 1 . Overall, a greater reduction was observed during experiment 2 when daylengths were shortest. Percent reduction during experiment 2 ranged from $75 \%$ to $90 \%$ under heavy shade, followed by $50 \%$ to $75 \%$ under moderate shade, and $29 \%$ to $60 \%$ in full sunlight. This more noticeable suppression of growth that occurred in late summer months is in agreement with Ervin and Koski (2001) who also reported greater growth reductions from TE in kentucky bluegrass in July and August as compared with May.

Although TE reduced leaf elongation rates, its application generally resulted in 
little additional benefit to those genotypes that already possessed good shade tolerance or dwarf growth habit. A collection week analysis was performed and determined that only a 4-week experiment $(r=0.98 ; P<$ 0.001 ) was necessary to achieve the same mean separation as the full experiment average (Table 8). Based on this analysis, it seems that leaf elongation rate is a useful parameter to identify hybrid bluegrasses with limited responses under moderate shade.

Regression analyses. Mean turfgrass quality ratings for each genotype in Table 2 were regressed against the calculated DLI for each light environment in Table 1 (Fig. 1). All coefficients of determination $\left(R^{2}\right)$ were $\geq 0.61$ with $P$ values ranging from 0.2408 to 0.0012 . The minimum DLI for each genotype was determined using the polynomial regression equations where $y=5$ (minimum acceptable turfgrass quality) (Bunnell et al., 2005). These results were similar to the mean quality performance of each genotype in experiment 1 (Table 2). 'Rebel Exeda' $\left(8.8 \mathrm{~mol} \cdot \mathrm{m}^{-2} \cdot \mathrm{d}^{-1}\right)$, 'DALBG 1201 ' $\left(0.8 \mathrm{~mol} \cdot \mathrm{m}^{-2} \cdot \mathrm{d}^{-1}\right)$ and TAES $5654\left(1.4 \mathrm{~mol} \cdot \mathrm{m}^{-2} \cdot \mathrm{d}^{-1}\right)$ had the lowest DLI requirements to maintain acceptable quality that fell within and below the calculated DLI range for heavy shade $(6.33 \pm 2.05$ and $4.08 \pm$ 1.63) (Table 1). Minimum DLI requirements for kentucky bluegrass CS\#4, and hybrid checks 'Thermal Blue' and 'Reveille',

Minimum Daily Light Integrals for Acceptable Turf Quality

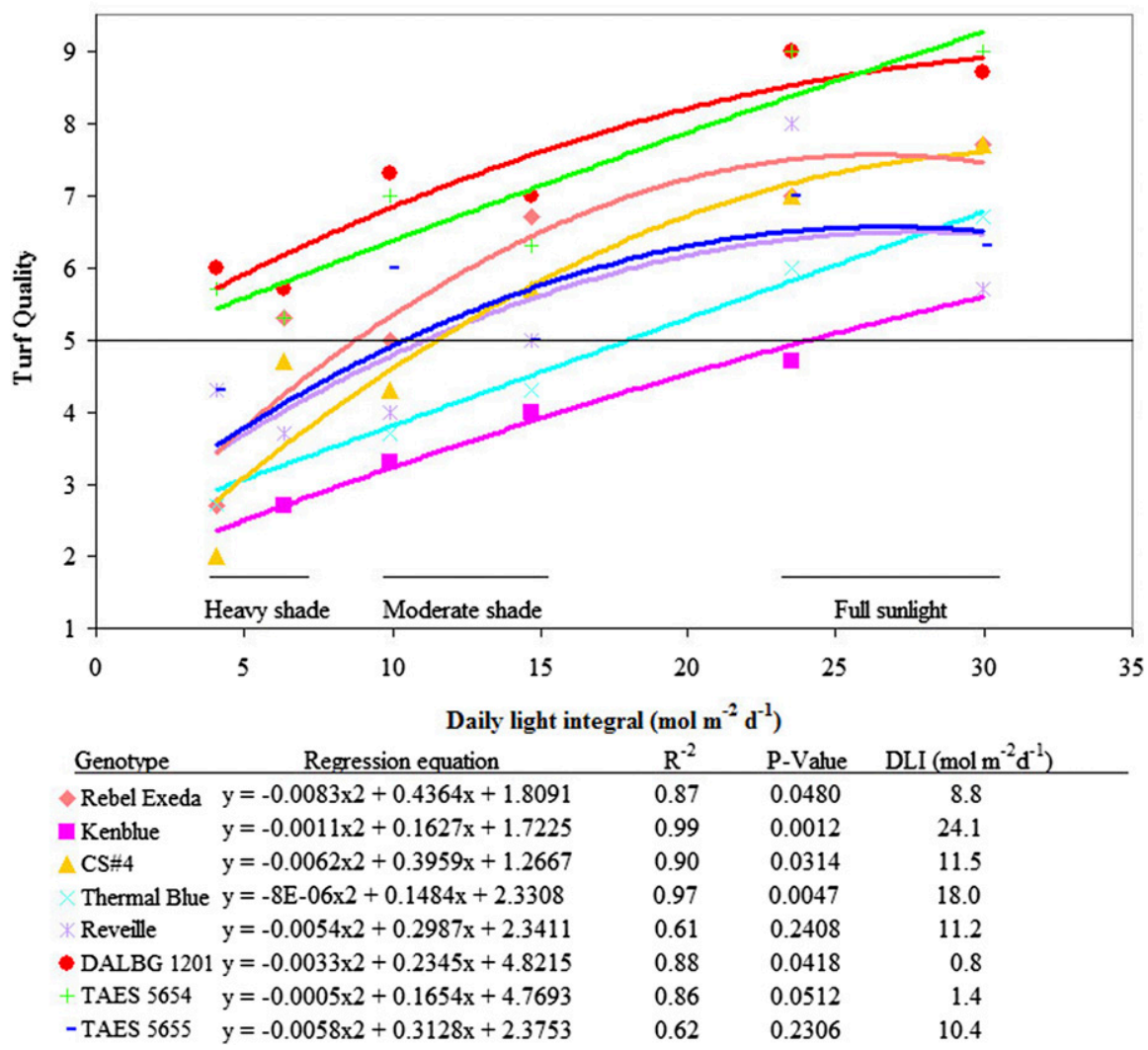

Fig. 1. Regression analysis determining the minimum required daily light integral (x) to achieve acceptable turfgrass quality $(y=5)$. Regressions were formed for each genotype using the experimental DLI from each light environment in Table 1, and the final turfgrass quality means from Table 2. Regression equations, coefficients of determination $\left(R^{2}\right), P$ values, and the calculated minimum DLI for each genotype are presented. suggested tolerance to moderate shade. The shade sensitivity of 'Kenblue' was supported by a high light requirement $\left(24.1 \mathrm{~mol} \cdot \mathrm{m}^{-2} \cdot \mathrm{d}^{-1}\right)$ equivalent to the calculated DLIs under full sunlight. These results suggest the selection of 'DALBG 1201' and TAES 5654 as alternatives to tall fescue for shaded environments.

Additional regressions were performed with leaf elongation rate for final turfgrass quality, final percent green cover, and cumulative clipping production (Table 9). Significant negative correlations were drawn for turfgrass quality and percent green cover under full sunlight and moderate shade (experiment 1). For cumulative clipping production, significant correlations were only observed under full sunlight.

Shade avoidance expressed as leaf elongation can cause leaf etiolation and reduced stand density over time (Beard, 1973) that directly impacts turfgrass quality and green cover. The significant negative correlation of leaf elongation rate with final turfgrass quality and cover in this study showed a similar decline as elongation rates increased. In this study, we found that moderate shade was a more optimum environment than heavy shade for screening genotypes using turfgrass quality and leaf elongation rates as selective measurements.

\section{Conclusions}

To our knowledge, this is the first documentation of comparative shade responses of texas bluegrass $\times$ kentucky bluegrass interspecific hybrids to reduced light environments, and in relation to tall fescue. Late spring months as well as moderate shade offered more favorable conditions for observing shade tolerances between hybrid bluegrasses and tall fescue compared with early fall. Leaf elongation rate was found to be a good predictor of shade tolerance in these genotypes. Furthermore, only 4 weeks were necessary to observe genotype differences using daily leaf elongation rate, suggesting that a relatively short period may be adequate to evaluate shade tolerance among experimental lines. Although TE effectively reduced leaf elongation rate in all environments, it did not improve turfgrass quality. Hybrid bluegrass genotypes ('DALBG 1201' and TAES 5654) exhibited lower DLI

Table 9. Correlations between leaf elongation rate and final percent green cover in both experiments under all shade environments.

\begin{tabular}{|c|c|c|c|c|c|c|c|}
\hline \multicolumn{8}{|c|}{ Correlation of traits } \\
\hline \multirow[b]{2}{*}{ Traits } & \multirow[b]{2}{*}{ Statistics } & \multicolumn{3}{|c|}{ Expt. 1} & \multicolumn{3}{|c|}{ Expt. 2} \\
\hline & & Full sun & Moderate shade & Heavy shade & Full sun & Moderate shade & Heavy shade \\
\hline Leaf elongation rate $\times$ final turfgrass quality ${ }^{z}$ & $r^{\mathrm{x}}$ & -0.78 & -0.74 & 0.00 & -0.90 & -0.65 & 0.00 \\
\hline \multirow[t]{2}{*}{ Leaf elongation rate $\times$ final green cover $(\%)^{z}$} & $\mathrm{r}^{\mathrm{x}}$ & -0.86 & -0.78 & -0.43 & -0.81 & -0.51 & 0.36 \\
\hline & $P$ value & 0.0059 & 0.0226 & 0.2815 & 0.0147 & 0.1946 & 0.4375 \\
\hline \multirow[t]{2}{*}{ Leaf elongation rate $\times$ clipping production $^{y}$} & $r^{x}$ & 0.75 & 0.09 & 0.66 & 0.89 & -0.57 & -0.16 \\
\hline & $P$ value & 0.0300 & 0.8400 & 0.0700 & 0.0000 & 0.1400 & 0.7100 \\
\hline
\end{tabular}

${ }^{z}$ The average leaf elongation rates calculated from week 10 were regressed against the averages for final turfgrass quality and final green cover for all genotypes. ${ }^{y}$ The overall average leaf elongation rates calculated across weeks $2,4,6,8$, and 10 were regressed against the average cumulative clipping production for all genotypes.

xPearson's correlation coefficient. 
requirements and may offer potential as an alternative to tall fescue for shaded areas in the transition zone or southern environments. Future field studies to evaluate the growth and developmental responses of hybrid bluegrasses to shade as well as evaluation of texas bluegrass under shade would be of value.

\section{Literature Cited}

Abraham, E.M., W.A. Meyer, S.A. Bonos, and B. Huang. 2008. Differential responses of hybrid bluegrass and Kentucky bluegrass to drought and heat stress. HortScience 43:2191-2195.

Beard, J.B. 1973. Turfgrass science and culture. Prentice Hall, Englewood Cliffs, NJ.

Beard, J.B. 1965. Factors in the adaptation of turfgrasses to shade. Agron. J. 57:457-459.

Brilman, L. 2009Kentucky bluegrass classification. Seed Res. of Oregon. Updated May 2012. 6 Jan. 2013. <http://www.sroseed.com/resources/ pdfs/articles/KB_classifications.pdf>.

Bunnell, B.T., L.B. McCarty, J.E. Faust, W.C. Bridges, Jr., and N.C. Rajapakse. 2005. Quanitifying a daily light integral requirement of a 'TifEagle' Bermudagrass golf green. Crop Sci. 45:569-574.

Burner, D.M. and C.P. West. 2010. Improving tall fescue shade tolerance: Identifying candidate genotypes. Agrofor. Syst. 79(1):39-45.

Busey, P. and E.H. Davis. 1991. Turfgrass in the shade environment. Proc. Fla. State Hort. Soc. 104:353-358.

Cockerham, S.T., S.B. Ries, G.H. Riechers, and V.A. Gibeault. 2002. Turfgrass growth response under restricted light: Growth chamber studies. In: California turfgrass culture. University of California Cooperative Extension 52(3-4): 13-20.

Ervin, E.H. and A.J. Koski. 2001. Kentucky bluegrass growth response to trinexapac-ethyl, traffic, and nitrogent. Crop Sci. 41:1871-1877.

Feldhake, C., J. Butler, and R. Danielson. 1985. Turfgrass evapotranspiration: Responses to shade preconditioning. Irrig. Sci. 6(4):265-270.

Gardner, D.S. and J.A. Taylor. 2002. Change over time in quality and cover of various turfgrass species and cultivars maintained in shade. HortTechnology 12:465-469.

Gardner, D.S. and B.G. Wherley. 2005. Growth response of three turfgrass species to nitrogen and trinexapac-ethyl in shade. HortScience 40:1911-1915.

Hall, M. 1996. Agronomy Facts: Kentucky bluegrasses. Penn. State Univ. Press, USA.

Hitchcock, A.S. 1950. Manual of the grasses of the United States (revised by A. Chase). U.S Gov't. Print Office, Washington, p. 1051.

Hurley, R. (n.d.). Turf-type tall fescues. 1 Jan. 2015 $<\mathrm{http}$ ://archive.lib.msu.edu/tic/mitgc/article/ 1989141.pdf>.

Karcher, D.E. and M.D. Richardson. 2005. Batch analysis of digital images to evaluate turfgrass characteristics. Crop Sci. 45:1536-1539.

Kephart, K.D. and D.R. Buxton. Nonstructural carbohydrates in cool- and warm-season perennial grasses adapted to shaded conditions. Research summaries. 1996. pp. 85-86.

Lickfeldt, D.W., D.S. Gardner, B.E. Branham, and T.B. Voigt. 2001. Implications of repeated trinexapac-ethyl applications on Kentucky bluegrass. Agron. J. 93:1164-1168.

Lin, C.H., R.L. McGraw, M.F. George, and H.E. Garrett. 1999. Shade effects on forage crops with potential in temperate agroforestry practices. Agrofor. Syst. 44:109-119.

Meeks, M., A. Chandra, S.P. Metz, A.D. Genovesi, J.C. Read, R.H. White, G. Miller, E. Guertal, H.W. Philley, and J. Sorochan. 2015. Registration of DALBG 1201 hybrid bluegrass. J. Plant Reg. 9:138-143.

Morris, K.N. 2010. National Kentucky bluegrass test-2005. Final Rpt. 2006-2010. NTEP no. 11-10.

Morris, K.N. 2013. National Kentucky bluegrass test - 2011. Prog. Rpt. 2013. NTEP no. 14-2.

Qian, Y.L. and M.C. Engelke. 1999. Influence of trinexapac-etyl on Diamond zoysiagrass in a shade environment. Crop Sci. 39:202-208.

Qian, Y.L., M.C. Engelke, M.J.V. Foster, and S.M. Reynolds. 1998. Trinexapac-ethyl restricts shoot growth and improves quality of $\mathrm{Di}$ amond zoysiagrass under shade. HortScience 33:1019-1022.

Richardson, M., E. Slack, T. Fermanian, S. Reid, E. Watkins, W. Meyer, B. Meyer, S. Langlois, M. Fraser, K. Hignight, and M. Goatley. 2010 Cooperative Turfgrass Breeders Test Report 2010. 21 Jan. 2015. <http://www.ctbt-us.info/>.

SAS Institute, Inc. 2012. JMP 10 for Windows. SAS Inst., Cary, NC.

Steinke, K. and J.C. Stier. 2003. Nitrogen selection and growth regulator applications for improving shaded turf performance. Crop Sci. 43:13991406.

Stier, J.C., J.N. Rogers, III, J.R. Crum, and P.E. Rieke. 1999. Flurprimidol effects on Kentucky bluegrass under reduced irradiance. Crop Sci. 39:1423-1430.

Stier, J.C. and J.N. Rogers, III. 2001. Trinexapacethyl and iron effects on supina and Kentucky bluegrasses under low irradiance. Crop Sci. 41:457-465.

Su, K., D.J. Bremer, S.J. Keeley, and J.D. Fry. 2007. Effects of high temperature and drought on a hybrid bluegrass compared with Kentucky bluegrass and tall fescue. Crop Sci. 47:2152-2161.

Tan, Z.G. and Y.L. Qian. 2003. Light intensity affects gibberellic acid content in Kentucky bluegrass. HortScience 38:113-116.

Tegg, R.S. and P.A. Lane. 2004. A comparison of the performance and growth of a range of turfgrass species under shade. Austral. J. Expt. Agr. 44:353-358.

Wallace, V., T. Fermanian, K. Blanchet, S. Ebdon, E. Watkins, W. Meyer, S. Langlois, M. Fraser, K. Hignight, and C. Fricker. 2013. Cooperative Turfgrass Breeders Test Report - 2013. 13 June 2014. <http://www.ctbt-us.info/>.

Wang, X.Y., T.M. Hu, Q.Z. Wang, L.M. Tian, X.L. Zhang, and K. Tian. 2009. Growth of Kentucky bluegrass as influenced by nitrogen and trinexapacethyl. Agr. Sci. China 8(12):1498-1502.

Watkins, E. and W.A. Meyer. 2004. Morphological characterization of turf-type tall fescue genotypes. HortScience 39:615-619.

Watson, V.H., G. Hagedorn, W.E. Knight, and H.A. Pearson. 1984. Shade tolerance of grass and legume germplasm for use in the southern forest range. J. Range Mgt. 37:229-232.

Wherley, B.G., D.S. Gardner, and J.D. Metzger 2005. Tall fescue photomorphogenesis as influenced by changes in the spectral composition and light intensity. Crop Sci. 45:562-568.

Wherley, B.G., A. Chandra, A. Genovesi, M. Kearns, T. Pepper, and J. Thomas. 2013. Developmental response of St. Augustinegrass cultivars and experimental lines in moderate and heavy shade. HortScience 48:1047-1051.

Wood, G.M. 1968. Evaluating turfgrasses for shade tolerance. Agron. J. 61:347-352.

Wu, L., D. Huff, and W.B. Davis. 1985. Tall fescue turf performance under a tree shade. HortScience 20:281-282. 